\title{
Grazing Incidence Metal Mirrors as the Final Elements in a Laser Driver for Inertial Confinement Fusion
}

\author{
Robert L. Bieri* \\ Michael W. Guinan \\ L-644, Lawrence Livermore National Laboratory \\ Livermore, CA 94551 \\ (415) 423-0897
}

\begin{abstract}
Grazing incidence metal mirrors (GIMMs) have been examined to replace dielectric mirrors for the fial elements in a laser beam line for an inertial confinement fusion reactor. For a laser driver with a wavelength from 250 to $500 \mathrm{~nm}$ in a 10 -ns pulse, irradiated mirrors made of $\mathrm{Al}, \mathrm{Al}$ alloys, or $\mathrm{Mg}$ were found to have calculated laser damage limits of $0.3-2.3 \mathrm{~J} / \mathrm{cm}^{2}$ of beam energy and neutron lifetime fluence limits of over $5 \times 10^{20}$ $14 \mathrm{MeV} \mathrm{n} / \mathrm{cm}^{2}$ (or 2.4 full porer years when used in a 1,000-MW reactor) when used at grazing incidence (an angle of incidence of 85 degrees) and operated at room temperature or at $77 \mathrm{~K}$. A final focusing system including mitrors made of Al alloy 7475 at room temperature or at liquid nitrogen temperatures used with B driver which delivers $5 \mathrm{MJ}$ of beam energy in 32 beams would reguire 32 mirsors of roughly $10 \mathrm{~m}^{2}$ each. This paper briefly reviews the methods used in calculating the damage limits for GMMMs and discusses critical issues relevant to tbe integrity and lifetime of such mirrors in a reactor environment.
\end{abstract}

\section{Introduction}

A 5-MJ laser producing a 1000-MJ thendonuclear yield will sesult in approximately $3.6 \times 10^{20} 14 \mathrm{MeV}$ neutrons per shot; at a distance of $50 \mathrm{~m}$ from the target this corresponds to a fluence of $1.1 \times 10^{12} \mathrm{n} / \mathrm{cm}^{2}$ for each shot. Since the last focusing or turning element of each beara cannot be shielded from these neutrons, it must be made of a matisial which can withstand the lifetime fluence associated with these radiation levels. Figures 1 and 2 diagram an inertial confinement fusion (ICF) laser driver whose use of metal mirrors[1] allows the more sensitive optical elements to be located out of dirc ct line-of-sight of the neutrons. In this geometry, the metal mirror is located $30 \mathrm{~m}$ from the target, and the dielectric mirror is $20 \mathrm{~m}$ behind the GIMM and
10 degrees removed from the direction of line-of-sight neutrons. Neution trarsport calculations indicate that this geometry provides the more sensitive optical components with a sufficiently reduced neutron rxposure to ensure an affordable component lifetime.

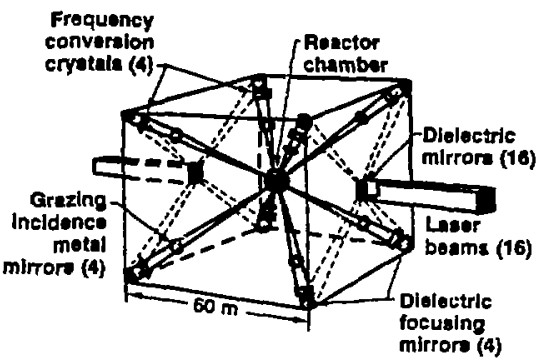

Figure 1. Spaceframe for an ICF reactor with a laser driver.

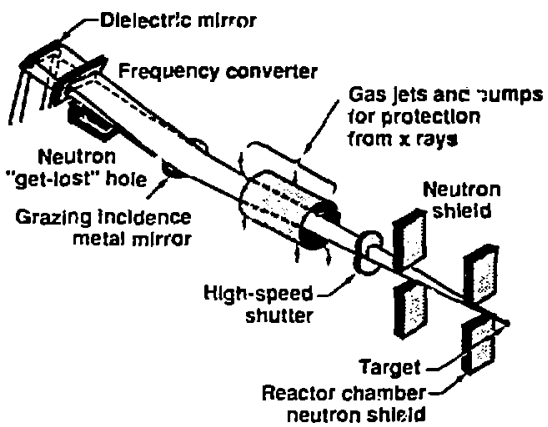

Figure 2. Location of final oplical elements for each beam line.

\footnotetext{
- MIT N.E. Graduate Student A STER MASTER 


\section{Protection of Sensitive Optics}

Neutron transport calculations have becu done to address several issues concerning how effectively the GIMMS shield the remaining optical elcments of the driver[2]. The calculations include the peutron flux, spectrum, and energs deposition for the GMM, the dielectric tus ning mirror, a KDP nonlinear optic crystal (if required for frequency conversion), liquid nitrogen to cool the mirror (if low temperature operation is required), and 1 Torr-meter of argon used to attenuate soft $x$-rays. The inplications for the activation of beam-line components were also ixamined.

\subsection{Effect of Neutrons on Multilayer Dielectric Mirrors}

Neutron radiation can destroy dielectric multilayer mirrors by degrading the optical transmission of the dielectric layers, by chemical decomposition of the dielectries, or by destroying the boundaries between layers. Transmission degradut ion data is sparse, but measure.nents[3] for $\mathrm{MgF}_{2}$ and $\mathrm{ZnS}$ show an order-of-magnitude degradation in absorption or transmission after $10^{16} \mathrm{n} / \mathrm{cm}^{2}$ (or about 1 hour of operation for an unshielded dielectric $50 \mathrm{~m}$ from a $1,000-\mathrm{MWe}$ reactor) for wavelengths of interest (250-500 nm). This damage may be removable through continunus annealing, and dielectrics may exist which bave color centers far removed from wavelengths of interest. Unfortunately, even if there is no loss of transmissive properties, the multilayer mirror can still be compromised by neutron damage. All ionic dielectrics will undergo significant radiolysis[4], radiationinduced chemical decomposition, after energy depositions of about $1 \mathrm{eV} /$ atom, or $5 \times 10^{17} \mathrm{n} / \mathrm{cm}^{2}$ for $\mathrm{MgF}_{2}$ i a few $\mathrm{eV} /$ atom energ: depostion is also enougb to cause signifiant amorphization in $\mathrm{SiO}_{2}$. For dielectric materials which are more resistant to danage, chemical mixing at the interfaces will still be a problem. Any collisional cascade at an interface will cause mixing of the two dielectrics and create a thio, possibly amorphiiss, third phase region with unknown optical properties. Collisional mixing at the boundaries will occur over a thickness of roughly $3 \mathrm{~nm} /(\mathrm{DPA})^{1 / 2}$ (1 DPA $\simeq 5 \times 10^{20} 14 \mathrm{MeV} \mathrm{n} / \mathrm{cm}^{2}$ for most dielectrics, which corresponds to about 1 full power year for a $1,000-\mathrm{MWe}$ plast), and enhanced diffusion will cause mixing over a thichness of roughly $30 \mathrm{sm} /(\mathrm{DPA})^{1 / 2}[5]$. If the very existence of a third pbase does not destroy the optical properties of a multilayer mirror, a change of thickness of only $\lambda / 16$ in a $\lambda / 4$ layer will destroy the constructive interference required for bigh refectivities. Under the most optimistic assumptions the best conceivable unslielded multilayer mirsor would probably only last a fraction of a full power year.

\subsection{Results of Neutron Transport Cal- culations}

\section{Neutron flus}

The results of the TART 3-D neutron transport code indicate that the neutron flux at the final optics is almost e ntiraly from ncutrons that shine directly on the GIMM. There will be significant contributions to the fiux at the firal turning mirror (and frequency converter if present) from scattered $14-\mathrm{MeV}$ neutrons and from lower energy backscattered neutrons; less than $10 \%$ of the neutrons arriving at the sensitive opties will have energies $\geq 1 \mathrm{MeV}$. There is little or no data on neutron damage to dielectric mirrors, but if we make the conservative assumption that a multilayes mirror with no color centers will have a lifetime fluence limit of $10^{17}--10^{\text {tg }}$ neutrons $/ \mathrm{cm}^{2}$ for neutrons with energies above $1 \mathrm{MeV}$, we can estimate the lifetime of a dielectric unirror. The total neutron flux at the turning mirror and the frequency converter, if present, is $1-2 \times$ $10^{10}$ aeutrons $/\left(\mathrm{cm}^{2} \mathrm{~s}\right)$. Since only $10 \%$ of these have energies above $1 \mathrm{MeV}$, the lifetime of the sensitive optics will be from 2-20 years. If there were no GIMM, the $f$ ux at $\mathrm{B}$ turning mirter $50 \mathrm{~m}$ from the target would be $10^{12} \mathrm{a} / \mathrm{cm}^{2}$ and consist almost entirely of bigh energy neutrons; this flux would give a lifetime of only 1 day under our damage assumptions.

\section{Activation}

Because of the activation of $\mathrm{Al}$ and $\mathrm{Mg}$, the mirrors will bave to be within a shielded area. A M $\mathrm{Mg}$ mirror would require 17 day's of cool-down before it could be worked on: an Al mirror would require remote or limited maintenance because of the long lived $A]^{26}$ that is formed after 10 days of operation. Waste disposal and accidental release are not a problem if the metal mirrors are replaced every 1-2 years.

\section{Laser Damage Thresholds for GIMMs}

\subsection{Reflectivities and Absorptances of Grazing Incidence Metal Mirrors}

The reflectivity of a conducting metal is a function of the wavelength and polarization of the incident light and the angle at which the light strikes the surfuce of the metal. Since laser drivers include polarization, we cais 
Table 1. Caiculated resistivitles, laser camage thresholcs, and mirror slzes for grazing Incldence metat mirrors.

\begin{tabular}{|c|c|c|c|c|c|c|c|c|}
\hline Metal & $\mathrm{Mg}$ & $\mathrm{Mg}$ & A) 1100 & Al1100 & A) 6061 & Al 6061 & Al 7475 & Al $\mathbf{7 4 7 5}$ \\
\hline $\begin{array}{l}\text { Oparating temp. (K) } \\
\text { Fatigue stress (MPa) } \\
\text { Surface temp. rise (K) } \\
\text { R (G.I.) } \\
\text { R (G.I.) atter irradiation } \\
\text { Max. beam energy (J/cm) } \\
\text { Min. miror area (m) }\end{array}$ & $\begin{array}{r}293 \\
34 \\
23 \\
0.9934 \\
0.9899 \\
0.34 \\
47\end{array}$ & $\begin{array}{r}77 \\
80 \\
75 \\
0.9941 \\
0.9900 \\
0.65 \\
24\end{array}$ & $\begin{array}{r}293 \\
43 \\
19 \\
0.9933 \\
0.9898 \\
0.3 ! \\
40\end{array}$ & $\begin{array}{r}77 \\
140 \\
90 \\
0.9940 \\
0.9899 \\
1.01 \\
15\end{array}$ & $\begin{array}{r}293 \\
180 \\
75 \\
0.9930 \\
0.9895 \\
1.29 \\
12\end{array}$ & $\begin{array}{r}77 \\
310 \\
158 \\
0.9937 \\
0.9896 \\
1.65 \\
9.4\end{array}$ & $\begin{array}{r}293 \\
290 \\
110 \\
0.9929 \\
0.9894 \\
1.88 \\
8.3\end{array}$ & $\begin{array}{r}77 \\
395 \\
185 \\
0.9936 \\
0.9895 \\
2.26 \\
6.9\end{array}$ \\
\hline
\end{tabular}

orient the mirrors so that the incident light hes the polavization (transverse electric) which proi.uces the highest refe -ivity. The reffection coefficient for a transverse electric (TE) polarized wave is given by [6]:

$$
r_{12}=\left(n_{1} \cos \theta_{1}-n_{2} \cos \theta_{3}\right) /\left(n_{2} \cos \theta_{1}+n_{2} \cos \theta_{2}\right)
$$

where $\theta_{1}$ is the angle or iscidence of the light in medium $i$. For a conductor. $n_{2} \cos \theta_{3}=u+i$, and the reflectivity is given by

$$
R=\left|r_{13}\right|^{2}=\frac{u^{2}+v^{2}-2 u \cos \theta_{1}+\cos ^{2} \theta_{1}}{u^{2}+v^{2}+2 u \cos \theta_{1}+\cos ^{2} \theta_{1}}
$$

for

$2 u^{2}=\left(n^{2}-k^{2}-\sin ^{2} \theta_{1}\right)+\left[\left(n^{2}-k^{2}-\sin ^{2} \theta_{1}\right)^{2}+4 n^{2} k^{2}\right]^{1 / 2}$,

$2 t^{2}=-\left(n^{2}-k^{2}-\sin ^{2} \theta_{1}\right)+\left[\left(n^{2}-k^{2}-\sin ^{2} \theta_{1}\right)^{2}+4 n^{2} k^{2}\right]^{1 / 2}$

where $n$ and $k$ are the frequency dependent refracture index and extinction coefficient of the metal.

The undarnaged grazing incidence refiectivities for Al and $\mathrm{Mg}$ calculated from experimental values for $n$ and $k$ for $250 \mathrm{~nm}$ light are shown in Table 1. The dependence of the grazing incidence reflectjvities on photon energy is shown in Figure 3.

\subsection{Radiation Damage to Mctal Mir- rors}

Successful operation of a magnesium or aluminum mirror located $30 \mathrm{~m}$ from a $1000 \mathrm{MJ}, 3-\mathrm{Hz}$ pellet is theoretically possible. The mirror must be shiclded from a particles, other charged particles, short-ranged neu-

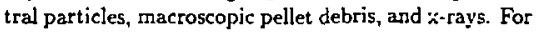
high $\rho R$ targets, less than one torr-meter of argon provides sufficient shielding for everything except the slowmoving pellet debris, which must be stopped by a highspeed shutter. Neutson darnage will increase the optical absorptance by a factor of at most two, thus lowering the laser-damage threshold by 'I factor of approximately two. Overall swelling, melting, vaporization, surface erosion, creep, and dimensional and mechanical instability, as well as increased absorptance from transmutation products and neutron-induced defects, are expected to be tolerable.

Neutron radiation can compromise the first mirror in three ways:

1) The laser damage threshold may be lowered by increased resistsivy of the metal dur to defects, transmutations, and surface roughenir $\mathrm{g}$ on an atomic scale.

2) The laser damage threshold may be lowered by increased absorptarice of the mirror due to micrascopic surface roughening.

3) The focusing of the mirror can deteriarate due to macroscopic distortions from sweiling or creep of the minor and support structure.

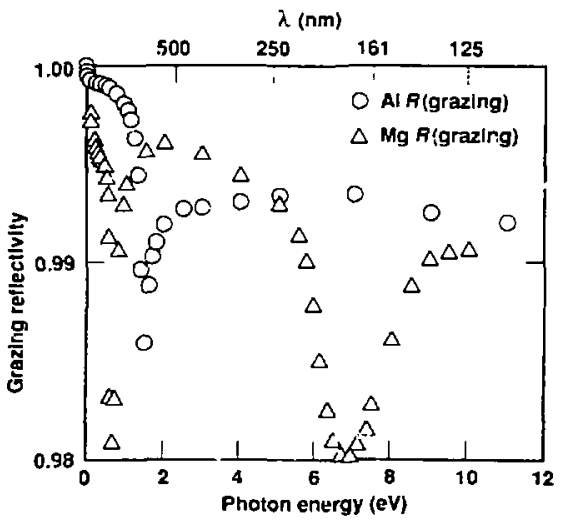

Figure 3. Grazing incidence reflectivity versus photon energy. 
The first effect aises the normal incidence absorptance by $1 \%$ and the grazing incidence ahorptance by $0.5 \%$ : in both cases a cont ribution to the absorpt ance of $0.35 \%$ is due to the rnomolo'ss skin effect resulting from roughening of the suriace on an atomic scale.

Damage from the second effect is negligible. The total sputtering from neutrons[7] ard scattered argon[8] will be only $30 \mathrm{~nm} /$ year, with the variance in the surface due to sputtering considerably smaller.

The third effect will be lifetime limiting for a roomtemperature mirror, but will not be a concern for a cryogenic mirror made of $\mathrm{Al}$ or $\mathrm{Mg}$. There will be negligible saturation swelling or creep in an $\mathrm{Al}$ or $\mathrm{Mg}$ mirror at cryogenic temperatures since the vacancies are immobile and saturation occurs. If the $7 \mathrm{~m} \times 1.8 \mathrm{~m}$ final mirror is conservatively limited to nonuniform swelling differences of $\lambda / 4$, the allowed volumetric swelling is $1.5 \%$ and the resulting lifetime fluence limits for $14-\mathrm{MeV}$ neutrons for room-temperature mirrors made of pure $\mathrm{Al}$, pure $\mathrm{Mg}$, and $\mathrm{Al} 7475$ are $2.5 \times 10^{20}, 8 \times 10^{19}$, and $5 \times 10^{20} \mathrm{a} / \mathrm{cm}^{2}$ respectively. The corresponding mirror lifetimes in a 1,000-MWe plant are 1.2. 0.4 , and 2.4 ful] power years.

\subsection{Laser Damage Limits for Irradiated Metal Mirrors}

The reflectivities for the damaged mirrors are used with thermal stress limits for a cyclic load to give the maximum aliowable beam energy density and corresponding minimum mirror size for each of 32 beams delivering a total of $5 \mathrm{MJ}$ of beam energs in $10 \mathrm{~ns}$. The resulting damage thresholds and mirror sizes are show'n in Table 1 .

\section{Critical Issues and Areas for Further Work}

From considerations of neutron damage, reflectivity, heat removal, and surface temperature rise, grazing incidence metal misors appear to be very attractive. The most crucial future work needed to verify the integrity of these mistrors includes:

- Experimental verification of laser damage thresholds

A small-scale experiment could be done with small undamaged mirrors to verify the calculated nor$\mathrm{mal}$ and grazing incidence reflectivities and to measure the effect of oxide coatings on reflectivity for each of the candidate metals. The effect of an oxidation layer on the normal incidence $353 \mathrm{~nm}$ reflectivity of $\mathrm{Al}$ or $\mathrm{Mg}$ is small (a frartion of a percent) or nonexistant, but the effect may be larger for grazing incidence. A L 's Alamos study of Al grazing incidence mirrors[9] showed signifcant degradation of the relectrvity for light with wavelengths below $100 \mathrm{~nm}$.

- Experiments with irradiated mirrors:

The laser damage experiments could be repeated with small irradiated samples to verify the effects of neutron irradiation on the laser oamage threshold. Although it may be difficult to get a sufficient source of $14 \mathrm{MeV}$ neutrons, fission reactor irradiations could be used and the results scaled with total darage energy.

- Protection of the final mirror from debris and $x$ rays

Even if the heat loads on a perfectly smooth and clean mirror are low enough to avoid surface vaporization, a particle or surface defect on the mirror surface would be exposed to the full normal incidence beam energy and could cause explosive "pitting" of the mirror surface. Accumulation of material (target debris, Flibe, or other coolant/brecder materials) from the reaction chamber on the mirror surface must be prevented. Gas jets used for protection from $x$-rays will not provide sufficient protection from high-velocity debris. There is a need for a high-specd mechasical shutter[10] to protect the mirtor. Both the gas and shutter systems require significant design work before their effectiveness can be judged.

- Development of techniques for cleaning the final mirrors

If a combination of high-speed shutters and gas jets can protect the mirror surface from ciamage but cannot keep it clez... it may be possible to remove accur ulated material from the mirror surface between shots. A possible technique would be to vaporize the contaminants with a lower energy beam between shots.

- Manufacturing studies for large high-quality mirrors

Large-scale high-quality mirrors can be manufactured, but the cost of producing $1\left(1 \cdot \mathrm{m}^{2}\right.$ mirrors with surfaces finished to 10 's of nm's inay be prohibitive.

Other concerns that are independent of future mirror work are: 
- The effect of large mirror cooling requirements on system efficiency

The energy required to cool a room-temperat ure mirros is not lase, but cooling a cryogenje mirror could require enough energy to significantly affect the plant power balance. Remoring $1 \%$ of the total beam power from the final mirrors at $77 \mathrm{~K}$ cculd require 10's to 100's of $\mathrm{AlW}$ s of recirculating pourer. Since low laser-driver efficiencies are a critical problem, the cost of additional recirculating power may be unacceptable.

- Nonuniform beam intensity

Nonuniform bean ixtensity could force the use of larger mirors. Multiplexed beams from KrF lasers will not only bave nopuniformities, they will also be coming from different angles of incidence. The best achievable peak-to-average power ratios at the final amplifier of Nova are typically 1.4-1.6. Streak camera profiles of a Nova beam inmediately after polarization and freguency conversion show a lower peak-to-average power ratio, with spike widths on the order of a millimeter. Transverse heat flow and stress release during a 10-ns shot in the mirror can only average out nonuniformities over distances of a ricron or less, so peaks wider than a micron will lead to local failure unless a larger mirror is used. Beam nonuniformity grow's as the beam converges, so nonuniformities at the mirror will be worse than those after the final focusing or conversion element.

- Brightness requirements for KrF lasers may require a minimurn solid angle illumination which would correspond to mirrors much larger than those which are damage limited. A required total illumination solid angle of $0 \mathrm{U} 4 \pi$ steradians would require a grazing incidence mirror size of $40 \mathrm{~m}^{2}$ for each miror in a 32-mirror systera.

\section{Conclusions}

Preliminary calculations indicate that grazing incidence metal mirrors appear to offer a solution to the critical problem of neutron damage to beam line components. A final mirror made of Al alloy could be used for 2.4 years at room temperature before replacement and for much longer times at $77 \mathrm{~K}$. Larger mirrors made of pure Al or $\mathrm{Mg}$ could also be used at $77 \mathrm{~K}$. Although there are important concerns which have yet to be investigated, spetal mirrors may provide a solution to the crucial problem of how to interface a laser driver with an ICF reactor. Solving this pro blem is essential if lasers are to be eredible driver candidates for ICF powet plants.

\section{Acknowledgements}

The authors would like to thank all of the people at Livermore who contributed time and help for our work. The list of contributors includes (but is certainly not limited to) Rajph Moir. Al Frank. Rich Vankonynenburg, Mike Tobin, Bill Hogan, and Lloyd Chise. One of the authors, R. Bieri. would also like to thank Dan Cohn and the Plasma Fusion Center at M.1.T. for supporting his mork. This work was carried out under the auspices of the U.S. Department of Energy by Lawrence Livermore National Laboratory under Contract W-7405-Eng48. 


\section{References}

(1j R. L. BIERI and M. W. GLINAX, An Analysis of Grazing Incideace Metal Mfirsors in a Laser ICF Reactor Drjver,"CCRL-JC-106094,Lawrence Livemore National Laboratory preprint (1990).

[2] M. TOBIN and R. BIERI. "Analysis of the Grazing Incidence Metal Mirror (GIMM) Concept for HYLIFE-IL" internal LLNL HYLIFE-I1 memo (1990).

(3) M. J. KEBER. Eandbook of Laser Science and Techrology. Vol. III, Optical Materials: Part 1, 336 and 366. CRC Press, Inc. (1986).

(4) F.W. CLINARD, JR., and L. W. HOBBS, "Radiation Effects in Non-Metals," in Physics of Radiation Efects in Crystals, 225-280, Eds. R.A. Johnson and A.X. Orlov, North Holland, Amsterdam (1956).

[5] H. WIDERSICH, "Phase Stability and Solute Segregation during Iradiation," jbid, 387-471.

[6] R. KLNGSLAKE. Ed., AF-? Optics and Opti. cal Engineering. Vol. 1I1, Optical Components, 317, Academic Press, N.Y. and London (1965). [i] O. li. HARLING and M.T. Thoxis in Pro. ceedings of the and Topical Mreting on Technol. ogy of Controlled Nuclear Fusion (USERDA Report CONF-760935-P1) Vol. 1, 149-167. (1976).

[8] H. H. ANDERSEN and H. L. BAY, in Sputter. ing by Particle Bombardment I, Topics in Applied Physicy, 17, ed. R. Behrisch, 167-168, SpringerVerlag, Berlin (298i).

[9] B.E. NE' NAM. "Multifreet Metal Mirror Design for Soft X-Rny and Extreme-liltraviolet FreeElectron Laser Resonators," in Laser Induced Dam. age in Optical Materials: 985, H.E. Bennett, A.H. Guenther, D. Mjlam, and B.E. Newnam, Eds., (Natl. Bur. Stand., Washington D.C.), NBS Spec. Publ. 746, 261-200, i988.

[10] D. G. NILSON and J. G. WOODivorTH, "F;nal Optic Protection Designs for ICE Containment Chambers," in Proceedings of the Eigth Topicul Meeting on The Technology of Fusion Encrgy. Salt Lake City, Utah (198S). 